\title{
Epidemiological aspects of fish in the diet
}

\author{
BY DAAN KROMHOUT \\ Division of Public Health Research, National Institute of Public Health and Environmental \\ Protection, PO Box 1, 3720 BA Bilthoven, The Netherlands
}

Fish consumption is not a characteristic of the diet of every European culture. In the thirteen cohorts of the five European countries that participate in the Seven Countries Study the fish consumption varied between no fish in Velika Krsna (Serbia) and an average of about $95 \mathrm{~g} / \mathrm{d}$ in Dalmatia (Croatia) (Kromhout et al. 1989). Even higher intakes were observed among fishermen in Japan $(250 \mathrm{~g} / \mathrm{d})$ and Eskimos who consumed $400 \mathrm{~g} \mathrm{seafood} / \mathrm{d}$. It has been shown that mortality from coronary heart disease is low among Eskimos and Japanese. Also the Dalmatian cohort of the Seven Countries Study is characterized by a low mortality rate from coronary heart disease (Menotti et al. 1993). It is attractive, therefore, to suggest that fish consumption may lower the risk for coronary heart disease.

Population comparisons in relation to diet-disease associations may be confounded by other dietary factors. The diets of the Eskimos, Japanese and Dalmatians are not only characterized by a high fish consumption but also by a relatively low intake of saturated fat. It may be hypothesized, therefore, that the low mortality rates for coronary heart disease can alternatively be explained by a low intake of saturated fat. Consequently prospective studies are needed to investigate the association between fish consumption and coronary heart disease mortality on the individual level.

In 1985 we published a paper showing an inverse relationship between fish consumption and 20-year mortality from coronary heart disease in the Zutphen Study, the Dutch contribution to the Seven Countries Study (Kromhout et al. 1985). This association was independent of other coronary heart disease risk factors. This inverse association was confirmed by the results of three other cohort studies: two from the USA, the Western Electric and the MRFIT study and by the Swedish Twin Study (Shekelle et al. 1985; Norell et al. 1986; Dolecek \& Grandits, 1991). Also in a case-control study carried out among Italian women an inverse relationship was found between fish consumption and myocardial infarction (Gramenzi et al. 1990). All those populations were characterized by a relatively low fish consumption, e.g. less than $50 \mathrm{~g}$ fish/d. In prospective studies carried out in cultures with a relatively high fish consumption pattern, e.g. coastal Norway and Hawaii no such association was found. This is possibly due to the fact that in these cultures a proper reference group, e.g. persons who did not consume fish, is lacking.

These results from observational studies are supported by the results of a secondary intervention trial carried out in Wales (Burr et al. 1989). A group of 2000 patients with a myocardial infarction were randomized into an experimental group and a control group. The patients in the experimental group got the advice to eat fatty fish at least three times weekly. Their fish consumption was four times as high as in the control group. After 2 years of follow-up a significantly lower mortality from coronary heart disease and from all causes was observed in the experimental group compared with the control group. Also these results support the hypothesis that a small amount of fish may protect against coronary heart disease. 
Eskimos have a much lower mortality rate from coronary heart disease compared with Danes but they have a higher mortality rate from stroke. The positive effect of a high consumption of seafood on coronary heart disease could be counteracted, therefore, by a higher mortality from stroke. We investigated the relationship between fish consumption and 15-year incidence of stroke in the Zutphen Study (S. O. Keli, personal communication). However, the incidence of stroke was inversely related to fish consumption. This may be due to the fact that most strokes in the Netherlands are of the thrombotic type while most strokes among the Eskimos are of the haemorrhagic type. Also these results support the hypothesis that a small amount of fish may protect against cardiovascular diseases.

If fish consumption is related to cardiovascular diseases the question is what mechanisms are responsible for this association. Male Eskimos have significantly lower triacylglycerols and total cholesterol but significantly higher high-density-lipoproteincholesterol levels compared with their Danish counterparts (Bang et al. 1971). In the Zutphen Study we found that men who habitually consumed about $30 \mathrm{~g} \mathrm{fish} / \mathrm{d}$ had significantly lower total and intermediate-density-lipoprotein-triacylglycerol levels compared with controls. The other lipoproteins fractions did not differ between groups.

Eskimos have significantly longer bleeding times and lower platelet counts compared with Danes (Dyerberg \& Bang, 1979). In the Zutphen Study such differences were not found between habitual fish consumers and controls. High amounts of fish oil, e.g. $5 \mathrm{~g} / \mathrm{d}$ have been shown to reduce blood pressure in intervention trials. Such an effect was not shown in trials using smaller dosages. Also in the Zutphen Study fish consumption was not associated with blood pressure in cross-sectional analysis.

Eskimos have significantly higher amounts of eicosapentaenoic acid (EPA) in the phospholipids compared with the Danes. EPA belongs to the class of $n-3$ polyunsaturated fatty acids that are present in fish. Habitual fish consumers in the Zutphen Study had also significantly higher concentration of EPA in the phospholipids compared with the controls. A higher concentration of EPA in the phospholipids may have a positive effect on membrane fluidity. Stiff membranes are a risk factor for atherosclerotic complications. A better membrane fluidity could play a role in the prevention of these complications.

It may be concluded that our knowledge of the mechanisms behind the inverse association between fish consumption and cardiovascular diseases is still fragmented. Therefore, mechanistic research should focus on the metabolic effects of a small amount of fish. However, the epidemiological evidence suggests that the consumption of one or two dishes of fish weekly may be of importance in the prevention of cardiovascular diseases.

\section{REFERENCES}

Bang, H. O., Dyerberg, J. \& Brondum Nielsen, A. (1971). Plasma lipid and lipoprotein pattern in Greenlandic west-coast Eskimos. Lancet i, 1143-1146.

Burr, M. L., Fehily, A. M., Gilbert, J. F., Rogers, S., Holliday, R. M., Sweetnam, P. M., Elwood, P. C. \& Deadman, N. M. (1989). Effect of change in fat, fish and fibre intakes on death and myocardial reinfarction: Diet and reinfarction (DART). Lancet ii, 757-760.

Dolecek, T. A. \& Grandits, G. (1991). Dietary polyunsaturated fatty acids and mortality in the Multiple Risk Factor Intervention Trial (MRFIT). World Review of Nutrition and Dietetics 66, 205-216.

Dyerberg, J. \& Bang, H. O. (1979). Haemostatic function and platelet polyunsaturated fatty acids in Eskimos. Lancet ii, 433-435. 
Gramenzi, A., Gentile, A., Fasoli, M., Negri, E., Parazzin, F. \& La Vecchia, C. (1990). Association between certain foods and risk of acute myocardial infarction in women. British Medical Journal 300, 771-773.

Kromhout, D., Bosschieter, E. B. \& De Lezenne Coulander, C. (1985). The inverse relation between fish consumption and 20-year mortality from coronary heart disease. New England Journal of Medicine 312, 1205-1209.

Kromhout, D., Keys, A., Aravanis, C., Buzina, R., Fidanza, F., Giampaoli, S., Jansen, A., Menotti, A., Nedeljkovic, S., Pekkarinen, M., Simic, B. S. \& Toshima, H. (1989). Food consumption patterns in the nineteen sixties in seven countries. American Journal of Clinical Nutrition 49, 889-894.

Menotti, A., Keys, A., Kromhout, D., Blackburn, H., Aravanis, C., Bloemberg, B., Buzina, R., Dontas, A., Fidanza, F., Giampaoli, S., Karvonen, M., Lanti, M., Mohacek, I., Nedeljkovic, S., Nissinen, A., Pekkanen, J., Punsar, S., Seccarecci, F. \& Toshima, H. (1993). Inter-cohort differences in coronary heart disease mortality in the 25-year follow-up of the Seven Countries Study. European Journal of Epidemiology (In the Press.)

Norell, S. E., Ahlbom, A., Feychting, M. \& Pedersen, N. L. (1986). Fish consumption and mortality from heart disease. British Medical Journal 293, 426.

Shekelle, R., Missell, L., Paul, O. \& MacMillan-Schryock, A. (1985). Fish consumption and mortality from coronary heart disease. New England Journal of Medicine 313, 820. 